\title{
Airway epithelial repair: breathtakingly quick and multipotentially pathogenic
}

\author{
J S Erjefält, C G A Persson
}

\begin{abstract}
Epithelial shedding, even to the point of airway denudation, had already been described as a common and unifying feature of asthma by the latter half of the 19th century. However, the repair processes that specifically follow the shedding-like loss of epithelial cells have only recently been examined in vivo. This paper discusses the exceedingly fast epithelial restitution and the potential pathogenic sequelae to epithelial shedding alone that have been unravelled. Epithelial cytoprotection emerges as an important property of future therapeutic drugs for the treatment of airways inflammatory conditions.
\end{abstract}

(Thorax 1997;52:1010-1012)

Keywords: asthma pathology, epithelial repair, mucosal barrier.

The centennial paradigm of epithelial shedding in asthma is based on findings of exfoliated epithelial cells in sputum and bronchoalveolar lavage (BAL) fluid and on microscopic observations of epithelial loss in necropsy and bronchial biopsy specimens. ${ }^{1-4}$ The occurrence of epithelial denudation has provided attractive explanations to phenomena such as nonspecific airway hyperresponsiveness. However, such causal links between epithelial damage and the pathophysiology of asthma are far from clear - for example, we still lack information about the extent and distribution of epithelial damage. Indeed, biopsy studies displaying up to $50 \%$ epithelial denudation ${ }^{23}$ have failed to demonstrate differences between asthmatic and healthy control subjects. Thus, the typical picture of denuded asthmatic bronchi probably involves artifacts inflicted during sampling and/ or sectioning. It has been suggested that the airways in patients with allergic diseases harbour a particularly fragile epithelium, ${ }^{45}$ based on exaggerated denudation at routine tissue handling. The maintenance of mucosal tightness in patients with eosinophilic airway disease $^{6}$ further indicates that a manifest epithelial denudation is too simplistic a description of the mucosa in desquamative diseases. In this paper we discuss recent observations which indicate that epithelial restitution starts in- stantaneously and proceeds at very high speeds after shedding and denudation. Furthermore, such shedding-repair processes may, by themselves, be partly responsible for the pathophysiology, the leucocyte pathology, and the remodelling that occur in desquamative airway diseases.

\section{Scientific basis}

HIGH SPEED RESTITUTION AFTER SELECTIVE LOSS OF COLUMNAR EPITHELIAL CELLS: A NEW ROLE FOR BASAL CELLS

Berkart suggested in $1889^{1}$ that exfoliation of mainly columnar cells is common in asthma. However, the capacity of basal cells to take over the barrier properties of the epithelium after a selective loss of columnar cells was only established quite recently. In intact human and animal airways it took less than 20 minutes for basal cells that remained after selective removal of the columnar epithelium to become extensively flattened and to cover the basement membrane. ${ }^{7}$ At the cell borders "seals" consisting of overlapping cytoplasmatic protrusions were formed. ${ }^{7}$ The same type of "seals" have also been observed in vivo at sites of epithelial damage induced by allergen challenge. ${ }^{5}$ The extreme readiness of basal cells to assume a barrier structure suggests that sacrifice of columnar cells may be regarded as a fully functioning airway mucosal defence mechanism. ${ }^{7}$ Releasing columnar cells and covering the basement membrane would thus be important roles for basal cells in airway defence and repair.

HIGH SPEED RESTITUTION AT EPITHELIAL DENUDATION

It is well known that repair of severely damaged and denuded airways eventually occurs via the migration of remaining neighbouring epithelial cells. ${ }^{8}$ However, there has been no information on the epithelial restitution that would occur after shedding-like denudation. Early repair events have been examined in some detail using a novel technique to create well defined epithelial denudation sites in vivo (without causing any bleeding or any damage to the basement membrane). ${ }^{9}$ Immediately after denudation ciliated and secretory epithelial cells at the 

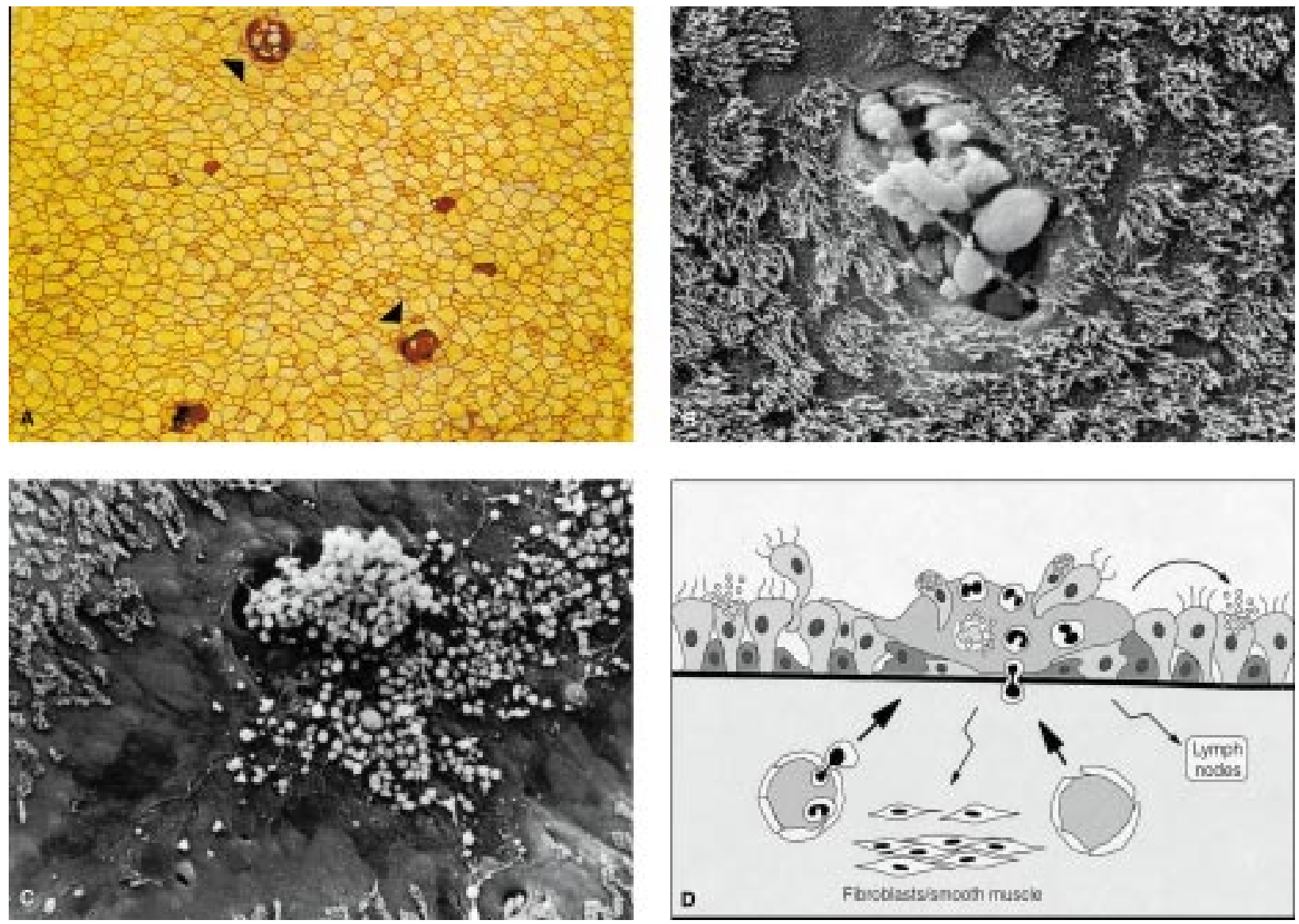

Figure 1 (A) Whole mount preparation, histochemically stained for epithelial cell borders, showing an overall view of sites of patchy epithelial damage (arrowheads). The detailed morphology of (B) small and (C) large damage sites is shown by scanning electron microscopy. (D) At sites of inflammation induced epithelial damage repair processes are immediately initiated; columnar cells (lightly shaded) and basal cells (darkly shaded) are involved in a continuous resealing of the epithelial lining. The restitution processes are aided by defence and growth promoting tissue responses - for example, extravasation of plasma and recruitment of leucocytes (thick arrows). Epithelial repair processes may also cause hypersecretion, proliferation of resident tissue cells, and increased local lymph node activity (thin arrows).

wound border dedifferentiate into repair cells that migrate rapidly until the denuded basement membrane has a new cellular cover. ${ }^{9}$ During the first few minutes after denudation the speed of migration is several $\mu \mathrm{m} / \mathrm{min}$. This acute repair occurs far more quickly than that seen in cell culture experiments, which indicates important roles for specific in vivo factors to the repair process (cell phenotypes, molecular milieu, dynamics, etc). ${ }^{9}$

TISSUE RESPONSES INDUCED BY EPITHELIAL SHEDDING

The epithelial denudation-restitution events are associated with many tissue responses including hypersecretion, ${ }^{9}$ plasma exudation, ${ }^{10}$ recruitment and activation of leucocytes, ${ }^{11}$ and increased proliferation of fibroblasts and smooth muscle cells. ${ }^{910}$

The mucosal microcirculation constitutes potentially the most important response organ during the early acute repair phase. Within minutes after denudation endothelial gaps are formed and a pronounced plasma exudation response is initiated. ${ }^{10} \mathrm{~A}$ gel-like network of fibrin and other attached plasma proteins is formed on the denuded basement membrane. ${ }^{10}$
Thus, the local extracellular milieu will promptly be endowed with plasma-derived molecules with defence and repair-promoting properties - for example, growth factors, cytokines, proteases, and antiproteases. ${ }^{10}$ Within a few minutes high numbers of leucocytes also accumulate in the denuded area. ${ }^{1011} \mathrm{In}$ terestingly, the epithelial damage-repair processes activate both eosinophils and neutrophils. ${ }^{10}$ Hence, tissue responses evoked by epithelial shedding alone may contribute significantly to the airway pathology in inflammatory diseases of the airway. ${ }^{9-11}$

EPITHELIAL DAMAGE-RESTITUTION IN ALLERGIC INFLAMMATION

Using cell border staining on whole mount preparations to get a good overall image we have recently examined the epithelial changes that occur during allergic inflammation in the large airways of guinea pigs (fig 1)..$^{5}$ The significant features to emerge from these studies are (1) the patchy distribution of epithelial damage, (2) instantaneous start of epithelial repair processes, and (3) focal localisation of inflammation to the injury-repair sites. 
Flattened epithelial repair cells were always present at the patches of injury, irrespective of the stage of epithelial damage. ${ }^{5}$ Hence, the sites of epithelial damage induced by inflammatory stimuli are exceedingly dynamic foci where epithelial shedding and repair processes occur simultaneously and where the basement membrane may be kept covered by poorly differentiated restitution cells (fig 1). The prompt start of epithelial repair also agrees with previous findings of a maintained airway absorption barrier in allergic airway diseases. ${ }^{6}$ Furthermore, sites of epithelial injury-repair in allergen-challenged airways are associated with a dense accumulation of plasma proteins and leucocytes including an abundance of extracellular eosinophil granules (the latter derived from cytolysis of eosinophils ${ }^{12}$ ).

\section{Therapeutic potential}

The notion that epithelial shedding-restitution processes alone are responsible for pathological changes in asthmatic airways offers new treatment options. Epithelial cytoprotective activity of future drugs would thus be expected to have beneficial effects on the exudative pathophysiology, granulocyte activation, and epithelial and subepithelial remodelling in patients with airway diseases.

\section{Conclusions}

The epithelial injury-repair processes discussed above suggest that the airway mucosa is well equipped for quick and efficient restitution, irrespective of the severity of epithelial shedding. Thus, in health and disease epithelial desquamation may occur without seriously im- pairing the mucosal barrier. Epithelial damagerestitution processes involve in vivo specific tissue responses which, besides promoting defence and repair, may contribute to several facets of the airway pathology observed in patients with inflammatory airway diseases. The prevention of epithelial damage may therefore become an important strategy in the development of new treatment options for allergic and inflammatory airway diseases.

This work was supported by the Swedish Medical Research Council projects 8308 and 4499, the Vardahl Foundation, and Astra Draco, Lund.

1 Persson CGA. Centennial notions of asthma as a desquamative, eosinophilic, exudative, and steroid-sensitive

2 Caroll N, Elliot J, Morton A, James A. The structure of large and small airways in nonfatal and fatal asthma. $A m$ Rev Respir Dis 1993;147:405-10.

3 Söderberg M, Hellström S, Sandström T, Lundgren R Bergh A. Structural characterization of bronchial mucosa biopsies from healthy volunteers: a light and electron microscopical study. Eur Respir F 1990;3:261-6.

4 Jeffery PK, Wardlaw AJ, Nelson FC, Collins JV, Kay AB Bronchial biopsies in asthma. An ultrastructural, quantitative study and correlation with hyperreactivity. Am Rev Respir Dis 1989;40:1745-53.

5 Erjefält JS, Korsgren M, Nilsson MC, Sundler F, Persson CGA. Prompt epithelial damage and restitution processes in allergen challenged guinea-pig trachea. Clin Exp Allergy $1997 ; 27$ (in press).

6 Persson CGA, Andersson M, Greiff L, Svensson C, Erjefält JS, Sundler F, et al. Airway permeability. Clin Exp Allergy 1995;23:807-14.

7 Erjefält JS, Sundler F, Persson CGA. Airway basal cells may promptly assume a barrier structure. Thorax 1997;52: 213-7.

8 Wilhelm DL. Regeneration of tracheal epithelium. F Pathol Bacteriol 1953;LXV:543-50.

9 Erjefält JS, Erjefält I, Sundler F, Persson CGA. In vivo restitution of the airway epithelium. Cell Tissue Res 1995; 281:305-16.

10 Persson CGA, Erjefält JS, Erjefält I, Korsgren M, Nilsson M, Sundler F. Epithelial shedding-restitution as a causative process in airway inflammation. Clin Exp Allergy 1996;26: $746-55$.

11 Erjefält JS, Sundler F, Persson CGA. Eosinophils, neutrophils, and venular gaps in the airway mucosa at epithelial phils, and venular gaps in the airway mucosa at epithelial 1666-74.

12 Persson CGA, Erjefält JS. Ultimate activation of eosinophils in vivo: lysis and release of clusters of free eosinophil granules (Cfegs). Thorax 1997;52:569-73. 